\title{
NATURAL POLYPHENOLS AS COMPOUNDS WITH BIOLOGICAL ACTIVITY*
}

\author{
VALENTIN I. POPA ${ }^{\mathrm{a}}$
}

\begin{abstract}
Plants produce thousands of phenolic and polyphenolic compounds as secondary metabolites. They are essential to the physiology of plants being involved in diverse functions such as lignification and structure, pigmentation, pollination, allelopathy, pathogen and predator resistance and growth. Approximately 8000 have been described to date, major classes being flavonoids, flavones, flavan-3ols, flavanones and anthocyanins. They can be isolated from biomass waste and cultivated and spontaneous plants by biorefining and used for their biological properties in different fields. In this paper some examples of uses such as: modulation agents of carbohydrates metabolism in diabetes and alcoholic fermentation, agents for regulation of physiological process in plants (germination, plant propagation and cultivation, tissue culture, bioremediation), modulation agents of metabolic processes in microorganisms (antifungal and antibacterial properties, antioxidants), models to study the influence of stress factors (hydrogen peroxide oxidation and UV irradiation and life span regulation), and carbon sources as substrates in medium cultivation of microorganisms, are presented.
\end{abstract}

Keywords: polyphenols, sources, isolation and characterization, biological properties

\section{INTRODUCTION}

Phenolic compounds posses an aromatic ring bearing one or more hydroxyl groups and their structures may range from that of a simple phenolic molecule to that of a complex high-molecular mass polymer. These compounds, one of the most widely occurring groups of phytochemicals, are of considerable physiological and morphological importance in plants. There are more than

\footnotetext{
This paper is dedicated to Professor Petre T. Frangopol, Honorary Member of the Romanian Academy, to remind us of the esteem in which this man is known for the fate of education and research in Romania.

a Technical University of lasi, Faculty of Chemical Engineering and Environmental Protection, Bulevardul Profesor Dimitrie Mangeron 73, Iași 700050, lasi, Romania, vipopa@tuiasi.ro
} 
8000 polyphenolic compounds identified, from which 4000 are flavonoids. For the polyphenols, the following properties were identified: antioxidant, prooxidant, anticancer agents, apoptosis-inducing, antibacterial, antiparasite, anti-HIV activities, amelioration of cardiovascular diseases, improvement of endothelial function, modulation of gamma-glutamylcysteine synthetase expression, improvement of health and survival on high fat diet, coloring agents, chelating agents, modulators of plant development. In the plants the phenolics may act as phytoalexins, antifeedants, attractants for pollinators, contributors to plant pigmentation, antioxidants and protective agents against UV light, amongst others. These bioactive properties made these compounds play an important role in plant growth and reproduction, providing an efficient protection against pathogens and predators, besides contributing to the colour and sensory characteristics of fruits and vegetables. In particular, natural phenols have been reported to have in particular, excellent properties as food preservatives as well as having an important role in the protection against a number of pathological disturbances, such as atherosclerosis, brain dysfunction and cancer. Many plant extracts and phytochemicals have been shown to have free radical scavenging properties, being known as antioxidants [1].

Interestingly, Denham Harman proposed The free radical theory of aging published in 1956 [2]. He proposed that free oxygen radicals produced in the body cause aging, and further stated that "reducing compounds" (what we would now call "antioxidants") might represent a "chemical means of prolonging effective life" and that these molecules "might be of benefit in the field of cancer chemotherapy and nutrition". At present, there is a lot of information regarding the role of natural compounds, such as polyphenols, which are tested in vitro and in vivo (animal, human, vegetable systems or microorganisms) to demonstrate their antioxidant properties [3].

The separation of polyphenols is carried out using an innovative technology proposed by us $[4,5]$. This technology allows separating the secondary and the main compounds of biomass, which can be used in different fields due to their biological activities $[6,7]$.

This paper presents the results obtained in the separation of polyphenols from different sources and some examples to use them for their biological activities.

\section{RESULTS AND DISCUSSION}

\section{Biological properties of polyphenols}

Concerning polyphenols, in our research programs, the following directions have been undertaken: modulation agents of carbohydrates metabolism in diabetes and alcoholic fermentation, agents for regulation of 
physiological process in plants (germination, plant propagation and cultivation, tissue culture, bioremediation), modulation agents of metabolic processes in microorganisms (antifungal and antibacterial properties, antioxidants), models to study the influence of stress factors (hydrogen peroxide oxidation and UV irradiation and life span regulation), and carbon sources as substrates in medium cultivation of microorganisms [8-26]. Polyphenols are large, complex molecules found almost exclusively in plant products. These molecules possess unique chemical characteristics, including the ability to powerfully scavenge reactive oxygen and nitrogen species, blocking oxidative damage that leads to inflammation and biological aging of tissue. Reactive oxygen species (ROS) and reactive nitrogen species (RNS), such as hydroxyl radical $(\cdot \mathrm{OH})$, hydrogen peroxide $(\mathrm{H} 2 \mathrm{O} 2)$, superoxide $(\mathrm{O} 2 \cdot-)$, nitric oxide $(\mathrm{NO} \bullet)$, peroxynitrite (ONOO-) and others are major sources of oxidative stress in cells, damaging proteins, lipids, and DNA. Oxidative DNA damage has been identified as a cause of cancer, aging and neurodegenerative diseases, such as Alzheimer's and Parkinson's, cardiovascular diseases, such as arteriosclerosis, and is the primary cause of cell death and tissue damage resulting from heart attack and stroke. Therefore, the prevention of oxidative stress caused by ROS and RNS has important implications for the prevention and treatment of diseases. LDL oxidation and platelet activation are two key events in atherogenesis, which lead to the formation of atherosclerotic lesion. These processes are interrelated, in that oxidized LDL can activate platelets, and activated platelets increase the susceptibility of LDL to oxidation. Atherosclerosis is associated with endothelial dysfunction, platelet activation, lipoprotein aggregation, macrophage foam cell formation, inflammation and thrombosis. These events are closely related and interact among themselves, finally leading to the formation of atherosclerotic lesions. Atherosclerosis involves both LDL oxidation and platelet formation and both processes can affect each other. Platelet activation is increased by oxidative stress, and oxidized LDL, in turn, was shown to enhance platelet activation. Activated platelets in turn can increase LDL oxidizability [27] any means of intervention that favorably affects the balance between the activity of the macrophage oxygenases and cellular antioxidants, as well as the elevation of LDL associated antioxidants or extrinsic antioxidants in plasma or extracellular space, can possibly contribute to the inhibition of the atherosclerotic process. Strategies to reduce $L D L$ oxidation thus involve the use of various antioxidants, which synergistically act on the cells and/or on the LDL molecules. In very simple terms, flavonoids inhibit the oxidation of polyunsaturated fatty acid groups in LDL by intercepting the free radical propagation of LDL oxidation. However, polyphenols are more than just free radical scavengers. Depending on their specific type and source, they also potently modulate gene expression. It means that polyphenols with unique characteristics can be chosen to address specific disease processes [28]. 
The common yeast Saccharomyces cerevisiae is often used in laboratory experiments as a model for understanding the biology of higher organisms due to the great similarity of basic life processes. The yeast is ideal in detecting cancerous chemicals, because its DNA-damage response system is remarkably similar to human cells. It can also be used to detect other types of damage, such as oxidative stress with a high degree of accuracy. Furthermore, the yeast cultures increased the expression of sirtuins, molecules known to trigger calorie restriction-like effects in multiple tissues, contributing to longevity. When polyphenols were added to yeast cell cultures, it was found that the treated cultures lived an average of 2-3 generations longer than the control cultures. Sirtuins are involved in a wide range of cellular processes, such as aging, transcription, apoptosis, inflammation and stress resistance, as well as energy efficiency and alertness during low-calorie situations. Sirtuins can also control circadian clocks and mitochondrial biogenesis. When yeast cultures were treated with polyphenols from grape seeds and spruce wood bark, it was observed that the treatment enhanced the cells' resistance to oxidative stress, both by inhibiting the production of harmful reactive oxygen species and by ramping up gene expression of vital natural cellular antioxidant systems. Thus, it was observed that polyphenols protect the yeast cell against hydrogen peroxide and UV irradiation and they can be used to inhibit cancer development. Grape seeds are also appreciated due to their content of phenolic compounds, such as gallic acid, catechin and epicatechin, and a wide variety of procyanidins (mainly condensed tannin). Their biological activities include: antioxidant properties and radioprotective effects, prevention of cataract, antihyperglycemic effects, enhancement of postprandial lipemia, modulation of the expression of antioxidant enzyme systems, improvement of insulin sensitivity and prevention of hypertriglyceridemia, inhibition of aromatase and suppression of aromatase expression, inhibition of protein kinase activity of epidermal growth factor receptor, protective effects against oxidative damage in mouse brain cells and anti-inflammatory effects. Oxidative stress is a condition arising from an imbalance between oxidant and antioxidants, leading to excess production of ROS. ROS are natural by-products of mithocondrial electrontransport chain reactions. The mithocondria are the "power plant" of the cell, generating chemical energy by producing adenosine triphosphate (ATP), the body's "energy currency" [29].

When polyphenols are used as agents for regulation of physiological process in plants (germination, plant propagation and cultivation, tissue culture, bioremediation), they could have similar effects with auxine and citokinine, and their action is synergetic with that of the hormones biosynthesized by plants. Polyphenolic extracts exhibited antibacterial properties against Staphylococcus aureus, Escherichia coli and Pseudomonas aeruginosa. 
The antimicrobial activity of the extracts against the tested microorganisms might be related to the presence of phenolic acids, such as gallic, protocatechuic, 3-hydroxybenzoic, chlorogenic acids and flavonoids, in the extracts [10]. Electrospun poly(2-hydroxyethyl methacrylate) (pHEMA) fibers, loaded with synthetic and natural antioxidants in the form of selected types of polyphenols, such as vanillic, gallic, syringic acids, catechin or natural spruce bark extract, were investigated with regard to their release behavior in terms of antioxidant activities [21,30]. At the same time it was developed poly(L-lactide-co-glycolide) (PLGA) bicomponent fibers, loaded with selected amounts of the catechin as natural polyphenolic antioxidant. The activity of the released catechin was assessed regarding its influence on multi-walled carbon nanotube (MWCNT) induced formation of reactive oxygen species (ROS) in the human alveolar epithelial cell line A549. As a proof of the concept, the activity of the released catechin in A549 cells stimulated with MWCNTs was determined, revealing a high reduction of ROS production in a dose dependent manner.

\section{CONCLUSIONS}

Polyphenolic compounds, along with other bioactive compounds, can be obtained from secondary resources or from cultivated plants using biorefining. There are numerous polyphenols with different structures and compositions, depending on the raw material and the techniques used for their extraction. Polyphenols are characterized by many interesting properties (antioxidant, antifungal and antibacterial, preventing and inhibiting agents of cancer, protection agents of the vascular system), which recommend their utilization in different biological systems. The biological properties of polyphenols have been demonstrated using different model systems: plant, animal and human cells and microorganisms. Polyphenols can be immobilized by electrospinning or included in different supports to be used in biological systems.

\section{EXPERIMENTAL}

\section{Isolation and characterization of polyphenols}

The separation of polyphenols can be carried out using a technology of biorefining that was previously proposed by us [4,5] some years ago. This innovative technology illustrated in Figure 1 allows separating the secondary and the main compounds of biomass, which can be further used in different fields due to their biological activities $[6,7]$. 
In our studies, for isolating polyphenols, we used wastes resulting from different industrial processes, as well as spontaneous and cultivated plants, as raw materials. Some of the raw materials we used are described below.

Spruce bark (Picea abies) is an abundant byproduct resulting from the wood processing industry. Spruce and pine barks are known by their rich content of proanthocyanidins and they have been used in folk medicine and as a dietary supplement.

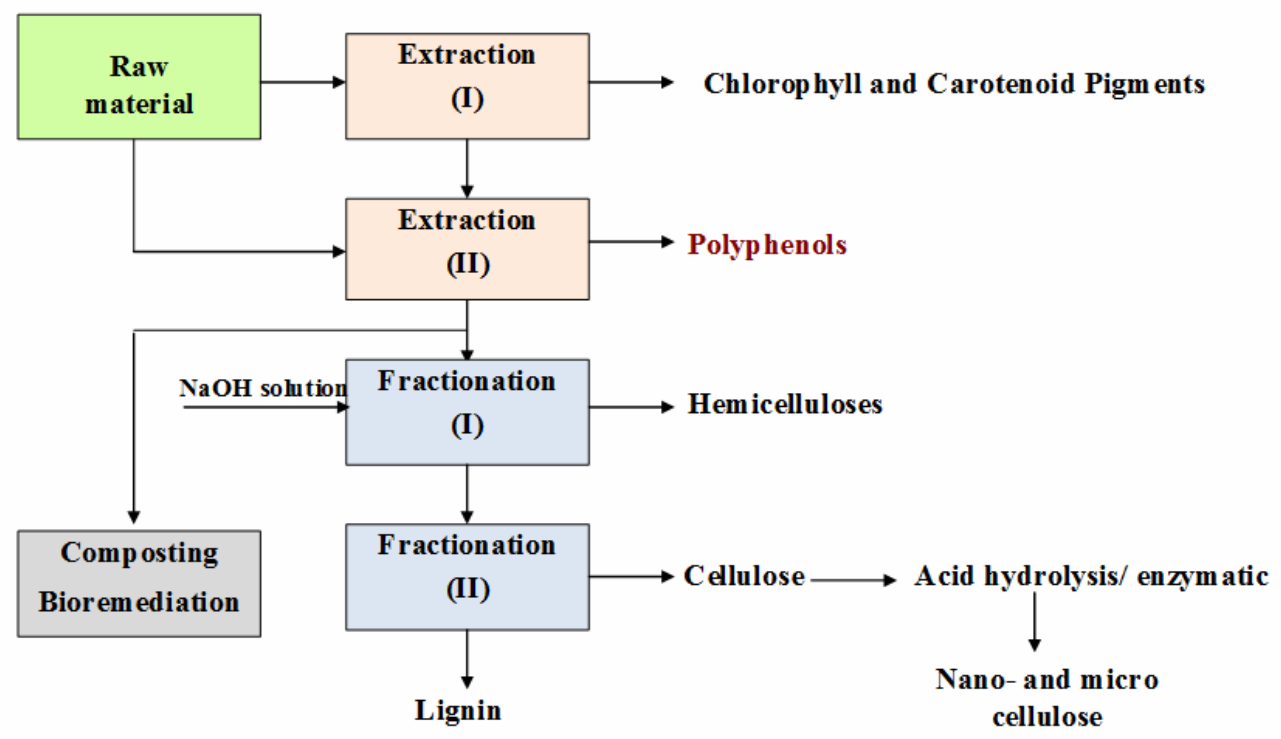

Figure 1. Separation of compounds with biological properties from biomass by biorefining

Grape seeds represent a considerable proportion of pomace, amounting to $40-50 \%$ on a dry matter. In recent years, naturally occurring plant phenolics in grape by-products have drawn a lot of interest, considering that grapes (Vitis vinifera) belong to the world's largest fruit crops. Grape seeds contain catechin, epicatechin, dimeric, trimeric and tetrameric proanthocyanidin, having antioxidant activity against reactive oxygen species (ROS). The grape seeds extract has proved helpful in various diseases, such as hepatic fibrosis, ischemia reperfusion injury (reducing the size of infarction in cardiac ischemia) and cancer. Also, it inhibits free radical production and has an important influence in the carbohydrate metabolism in fermentation and diabetes. 
Hawthorn (Crategus monogyna) is a spontaneous plant known in traditional medicine for its many health benefits, especially in cardiovascular health.

Asclepias syriaca was selected and cultivated in our studies due to its energetic value, being rich in hydrocarbons. It is characterized by a complex composition comprising cellulose, hemicelluloses, lignin, polyphenols, sugars, alkaloids and hydrocarbons. It can be used as raw material to be processed by the biorefining technology proposed by us, allowing recovering all the compounds [4]. At the same time, hydrocarbons and unsaturated fatty acids could be used as an alternative to obtain triglycerides and biofuel.

Lingonberry (Vaccinium myrtillus L.) is a wild shrub, whose fruits and aerial parts are used as food and in traditional medicine, due to their richness in bioactive phenolic compounds. Seasonal variations of the phenolic constituents in bilberry leaves, stems and fruits have been observed and characterized from the viewpoint of antioxidant properties. The extraction of polyphenols can be performed using different techniques and solvents, which influence the composition and yields of the recovered compounds. Thus, the following techniques have been used: conventional Soxhlet extraction, ultrasounds, microwave and supercritical fluids assisted extractions. Water, methanol and ethanol have been used as extraction agents and their influence on the extracted total polyphenolic compounds (TPC) was determined by the Folin-Ciocalteu method. The content in phenolic compounds was correlated well with TPC and the 2,2-diphenyl-1-picrylhydrazyl (DPPH) radical scavenging activity. The total phenolic content was expressed in $\mathrm{mg}$ gallic acid equivalents (GAE) per g dried extract (d.e.), using a standard curve of gallic acid (0.1$2.0 \mu \mathrm{g} / \mathrm{mL}$ ). The information concerning the polyphenol compositions was enhanced when we used HPLC associated with mass spectroscopy. At the same time, after alkaline hydrolysis, other polyphenolic acids were identified, which proved that some of them are present in glycoside form.

\section{REFERENCES}

1. I. Ignat; I. Volf; V. I. Popa; Food Chem., 2011, 126, 1821-1835

2. D. Harman; J. Gerontol., 1956, 11(3), 298-300

3. I. Volf; V. I. Popa; Integrated Processing of Biomass Resources for Fine Chemical Obtaining: Polyphenols. In Biomass as Renewable Raw Material to Obtain Bioproducts of High-Tech Value; V. Popa, I. Volf, Eds.; Elsevier: Radarweg, Netherlands, 2018, Chapter 4, pp. 113-160

4. Cr. I. Simionescu; V. Rusan; V. I. Popa; Cellulose Chem. Technol., 1987, 21(1), $3-16$

5. O. C. Bujor; I. A. Talmaciu; I. Volf; V. I. Popa; Tappi J., 2015, 14(3), 187-193 
6. V. I. Popa; Hemicelluloses in Pharmacy and Medicine. In Polysaccharides in medicinal and pharmaceutical applications, V. Popa Ed.; Smithers Rapra, London, UK, 2011, Chapter 2, pp.57-88

7. V. I. Popa; Lignin in Biological System, in Polymeric Biomaterials, vol.1, Structure and function; S. Dumitriu, V. Popa, Eds., CRC Press/Taylor \& Francis, NW, USA, 2013, Chapter 21, pp.709-738.

8. A.-R. Hainal; I. Ignat; I. Volf; V. I. Popa; Cellulose Chem. Technol., 2011, 45(3-4), 211-219

9. A.-R. Hainal; R. Diaconescu; I. Volf; V. I. Popa; Rom. Biotechnol. Lett., 2012, 17(2), 7084-7092

10. I. Ignat; D. G. Radu; I. Volf; A. I. Pag ; V. I. Popa ; Cellulose Chem. Technol., 2013, 47(5-6), 387-399

11. C. Tanase; I. Volf; S. Vintu; R. Gradinaru; V. I. Popa; Cellulose Chem. Technol., 2013, 47(7-8), 553-563

12. I. Volf; I. Ignat; M. Neamtu; V. I. Popa; Chem. Pap., 2014, 68(1), 121-129

13. C. Tanase; I. Boz; A. Stingu; I. Volf; V. I. Popa; Ind. Crop. Prod., 2014, 60, 160-167

14. R.-E. Ghitescu; A.-M. Popa; V. I. Popa; R. M. Rossi; G. Fortunato; Int. J. Pharm., 2015, 494, 278-287

15. R.-E. Ghitescu; I. Volf, C; Carausu; A.-M. Bühlmann; I. A. Gilca; V. I.Popa; Ultrason. Sonochem., 2015, 22, 535-541

16. A. I. Talmaciu, I. Volf, V. I. Popa, Chem. Biodivers., 2015, 12, 1635-1651

17. L. Lazar; A. I. Talmaciu; I. Volf; V. I. Popa; Ultrason. Sonochem., 2016, 32, 191-197

18. C. Tanase; I. Boz; V. I. Popa; Rom. Biotechnol. Lett., 2016, 21(1), 11238-11245

19. A. I. Talmaciu; M. Ravber; I. Volf; Z. Knez; V. I. Popa; J. Supercrit. Fluid., 2016, $117,243-251$

20. C. Tanase; S. Oroian; S.-L. Cosarca; V. I. Popa; Cellulose Chem. Technol., 2016, 50(5-6), 529-534

21. R.-E. Ghitescu; A.-M. Popa; A. Schipanski; C. Hirsch; G. Yazgan; V.I. Popa; R. M. Rossi; K. Maniura-Weber; G. Fortunato; Eur. J. Pharm. Biopharm., 2018, 122, 78-86

22. O.-C. Bujor; C. Le Bourvellec; I. Volf; V. I. Popa; C. Dufour; Food Chem., 2016, $213,58-68$

23. O.-C. Bujor; C. Giniès; V. I. Popa; C. Dufour; Food Chem., 2018, 252, 356-365

24. C. Tanase; A. I. Talmaciu; C. I. Bara; I. Boz; I. Volf; V. I. Popa; BioResources, 2018, 13(2), 3994-4007

25. C. Tanase; L. Berta; A. Mare; A. Man; A. I. Talmaciu; I. Roșca; E. Mircia; I. Volf; V. I. Popa; Eur. J. Wood Wood Prod., 2020, 78, 281-291

26. D. Jitaru (Ciobotariu); D. Ungureanu; M. Ciocoiu; M. Badescu; V. I. Popa; Rev. Med. Chir. Soc. Med. Nat., lasi, 2005, 109, supl. nr. 1, 33-37

27. M. Aviram; Eur. J. Clin. Chem. Clin. Biochem., 1996, 34, 599-608

28. K. S. Panikar; R. A. Anderson; Int. J. Mol. Sci., 2011,12, 8181-8207

29. S. Davinelli; N. Sapere; M. Visentin; D. Zella; G. Scapagini; Immunity \& Ageing, 2013, 10, 28 (5 pages)

30. R.-E. Ghitescu; A.-M. Popa; V. I. Popa; R. M. Rossi; G. Fortunato; Intern. J. Pharm, 2015, 494, 278-287 